\title{
Efeito da contraceção com etinilestradiol em alta dose na densidade mineral óssea em adolescentes: qual a evidência?
}

Juliana Silva Castro, ${ }^{1}$ Bruna Tavares, ${ }^{2}$ Marta Guedes ${ }^{3}$

\section{RESUMO}

Objetivos: Os contracetivos hormonais combinados $(\mathrm{CHC})$ são o método de contraceção hormonal mais utilizado na adolescência. Estes levam a níveis de estradiol inferiores aos fisiológicos, que poderão influenciar a aquisição do pico de densidade mineral óssea (DMO). O objetivo deste estudo foi determinar o impacto na DMO da contraceção hormonal combinada por via oral com 30-35 $\mu$ g de etinilestradiol (EE) em comparação com placebo ou doses inferiores de EE a $30 \mu \mathrm{g}$ em adolescentes saudáveis dos 10-19 anos. Fontes de dados: National Guideline Clearinghouse, National Institute for Health and Care Excellence Guidelines Finder, Canadian Medical Association Practice Guidelines Infobase, The Cochrane Library, Database ofAbstracts of Reviews of Effectiveness, Bandolier, Evidence Based Medicine Online e MEDLINE.

Métodos de revisão: Foram pesquisadas normas de orientação clínica, ensaios clínicos aleatorizados e controlados, revisões sistemáticas e meta-análises, publicados entre março/2007 e março/2017, nas línguas portuguesa, espanhola, inglesa e francesa. Os termos MeSH utilizados foram: adolescent, ethinyl estradiol e bone density. Para estratificar o nível de evidência e a força de recomendação foi utilizada a Strenght of Recommendation Taxonomy, da American Academy of Family Physicians.

Resultados: Identificaram-se 76 publicações, das quais foram incluídas cinco: dois ensaios clínicos aleatorizados e controlados, uma revisão sistemática, um estudo de coorte e uma guideline. Os primeiros mostram que as adolescentes não utilizadoras de $\mathrm{CHC}$, independentemente das doses, têm DMO mais elevadas do que as usuárias. A DMO das adolescentes que utilizam etinilestradiol em doses altas (30-35 $\mu \mathrm{g}$ ) parece ser menos afetada em comparação com as que o utilizam doses inferiores (<30 $\mu \mathrm{g})$. Na revisão sistemática incluída foram encontrados dados discordantes com um dos estudos, que referia não haver diferenças entre as várias doses de etinilestradiol. O estudo de coorte incluído revelou um aumento de DMO nas adolescentes com 30-35 $\mu$ g de etinilestradiol até aos 24 meses de toma. Entre os 24 e os 36 meses verificou-se uma redução da aquisição de DMO nas usuárias de doses mais altas de EE comparativamente com as usuárias de doses mais baixas. A guideline da Faculty of Sexual and Reproductive Healthcare refere que a dosagem parece não afetar a DMO.

Conclusão: Foi atribuída a esta revisão uma força de recomendação B, para o uso de $\mathrm{CHC}$ com etinilestradiol na dose igual ou superior a $30 \mu \mathrm{g}$, em adolescentes, para um menor impacto na DMO.

Palavras-chave: Adolescentes; Etinilestradiol; Densidade mineral óssea.

\section{INTRODUÇÃO}

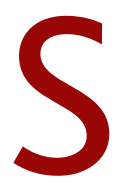
egundo a Organização Mundial da Saúde, a adolescência é o período de transição entre a infância e a vida adulta que compreende as idades entre os dez e os dezanove anos e se caracteriza pelos impulsos do desenvolvimento físico, mental, emocional, sexual e social. ${ }^{1}$ É na adolescência que a maioria dos jovens inicia a atividade sexual e, por conseguinte, inicia um método contracetivo. ${ }^{2}$ Os contra- cetivos hormonais combinados (CHC) administrados por via oral são o método hormonal mais frequentemente utilizado nesta faixa etária. ${ }^{3}$

1. Médica Interna de Medicina Geral e Familiar. USF Terras de Santa Maria, ACeS Entre Douro e Vouga I.

2. Médica Interna de Medicina Geral e Familiar. USF Vale do Vouga, ACeS Entre Douro e Vouga II.

3. Médica Interna de Medicina Geral e Familiar. USF Entre Margens, ACeS Entre Douro e Vouga II. 
O pico de massa óssea é influenciado por diversos fatores, entre eles: idade, peso, altura, idade da menarca, atividade física, dieta, genética, tabagismo e hormonas esteroides endógenas e exógenas. ${ }^{4}$ Este último fator influencia particularmente o metabolismo ósseo. Nas adolescentes e mulheres jovens, o estradiol endógeno diminui a atividade osteoclástica e exibe um efeito neutro sobre os osteoblastos, conduzindo assim a uma adequada mineralização. ${ }^{5}$

A densidade mineral óssea (DMO) aumenta rapidamente com o desenvolvimento pubertário a partir dos onze anos. ${ }^{6}$ A mineralização máxima do colo do fémur é alcançada aproximadamente aos dezasseis anos. Na coluna lombar, 95\% do pico de DMO é alcançado nos três anos após a menarca, sendo que, desses, $47 \%$ são atingidas um ano antes da menarca. ${ }^{7-8} \mathrm{Nas} \mathrm{mu}-$ lheres jovens saudáveis, o aumento de massa óssea na coluna lombar continua durante a terceira década de vida, terminando por volta dos trinta anos..$^{7-8} \mathrm{Os}$ CHC per os levam a níveis de estradiol inferiores aos fisiológicos, que poderão influenciar negativamente a aquisição do pico de DMO em adolescentes.

Num estudo observacional realizado em três unidades de saúde familiar da região norte de Portugal, com cerca de 400 adolescentes, os autores verificaram que a maioria utilizava como método contracetivo os $\mathrm{CHC}$ administrados por via oral de baixa dose (15 ou $20 \mu \mathrm{g}) .^{12}$

Esta revisão tem como objetivo determinar, à luz de evidência atual, qual o impacto na densidade mineral óssea dos contracetivos hormonais combinados per os de 30-35 $\mu$ g de etinilestradiol, em comparação com placebo ou contracetivos hormonais combinados com doses inferiores de etinilestradiol nas adolescentes saudáveis.

\section{MÉTODOS}

Foi realizada uma pesquisa de normas de orientação clínica (NOC), ensaios clínicos aleatorizados e controlados (ECAC), revisões sistemáticas (RS) e meta-análises (MA), publicados entre março de 2007 e março de 2017, nas línguas portuguesa, espanhola, inglesa e francesa, indexados nas bases de dados da National Guideline Clearinghouse, National Institute for Health and Care Excellence (NICE), Guidelines Finder, Canadian Medical Medical, Association Practice Guidelines Infobase,
The Cochrane Library, Database of Abstracts of Reviews of Effectiveness (DARE), Bandolier, Evidence Based Medicine Online e MEDLINE, utilizando os termos MeSH 'adolescent', 'ethinyl estradiol'e 'bone density'e os termos DeCS 'adolescente', 'etinilestradiol'e 'Densidade óssea'.

Os critérios utilizados para a inclusão dos artigos consistiram em: população-alvo constituída por adolescentes saudáveis do sexo feminino dos 10-19 anos; intervenção terapêutica com 30-35 $\mu$ g de etinilestradiol (EE) via oral (contracetivo hormonal combinado, sem restrições do tipo e dosagem de progestativo utilizado); comparação com placebo ou contracetivo hormonal combinado utilizando doses inferiores a $30 \mu \mathrm{g}$ etinilestradiol; e outcome relacionado com o impacto na densidade mineral óssea (DMO).

Foram utilizados, como critérios de exclusão, estudos não-randomizados, artigos duplicados, artigos de opinião, artigos de revisão clássica de tema e artigos discordantes do objetivo da revisão. Foram também excluídos ensaios clínicos incluídos em RS ou MA selecionadas (Figura 1).

Para estratificar o nível de evidência (NE) dos estudos e a força de recomendação (FR) foi utilizada a Strenght of Recommendation Taxonomy (SORT), da American Academy of Family Physicians.

A seleção dos artigos para revisão foi feita em triplicado pelas três autoras que, perante dúvidas, discuti- 


\begin{tabular}{|l|c|c|}
\hline $\begin{array}{l}\text { QUADRO I. Guidelines } \\
\text { Referência }\end{array}$ & \multicolumn{1}{|c|}{ Recomendação } \\
\hline $\begin{array}{l}\text { Guidelines: Faculty of Sexual and } \\
\begin{array}{l}\text { Reproductive Healthcare. } \\
\text { Reino Unido (2010) }\end{array}\end{array}$ & "Os CHC parecem não afetar a DMO (...)" & 3 \\
\hline
\end{tabular}

Legenda: $\mathrm{DMO}=$ densidade mineral óssea; $\mathrm{CHC}$ = contracetivo hormonal combinado; $\mathrm{NE}=$ nível de evidência. as jovens entre os 16 e os 19 anos que efetuaram a dose mais baixa de EE (15 $\mu \mathrm{g} v s 30 \mathrm{~g}$ ) tiveram tendencialmente menor DMO.

O estudo de Scholes e colaboradores, de 2010, incluiu 301 adolescentes com idades compreendidas entre os 14 e os 18 anos, divididas em três grupos: CHC na dose $<30 \mu \mathrm{g}$

\section{QUADRO II. Revisão sistemática}

\begin{tabular}{|c|c|c|c|c|c|}
\hline \multicolumn{2}{|c|}{ Referência } & \multirow{2}{*}{$\begin{array}{l}\text { População } \\
\text { Adolescentes } \\
\text { 16-19 anos } \\
(n=75)\end{array}$} & \multirow{2}{*}{\begin{tabular}{l}
\multicolumn{1}{c}{ Intervenção } \\
$n=65$ com $\mathrm{CHC}$ \\
Divididas em 2 grupos: $\mathrm{EE}$ \\
$30 \mu \mathrm{g}$ vs $\mathrm{EE} 15 \mu \mathrm{g}$ \\
$n=10$ sem $\mathrm{CHC}$ \\
Seguimento de um ano
\end{tabular}} & \multirow{2}{*}{\begin{tabular}{l}
\multicolumn{1}{c}{ Resultados } \\
Adolescentes que tomaram a \\
dose mais baixa de EE têm \\
tendencionalmente menor DMO
\end{tabular}} & \multirow{2}{*}{$\frac{\mathrm{NE}}{2}$} \\
\hline $\begin{array}{l}\text { Ziglar e Hunter } \\
\text { (2015) }\end{array}$ & $\begin{array}{l}\text { - Lattakova, et al. } \\
\text { (2009) }\end{array}$ & & & & \\
\hline & $\begin{array}{l}\text { - Scholes, et al. } \\
\text { (2010) }\end{array}$ & $\begin{array}{l}\text { Adolescentes } \\
14-18 \text { anos } \\
(n=301)\end{array}$ & $\begin{array}{l}n=73: \text { CHC com EE }<30 \mu g \\
n=121: \text { CHC com EE } 30-35 \mu g \\
n=107 \text { sem } C H C\end{array}$ & $\begin{array}{l}\text { A DMO não foi } \\
\text { significativamente diferente na } \\
\text { coluna lombar ou fémur nos três } \\
\text { grupos }\end{array}$ & \\
\hline
\end{tabular}

Legenda: $\mathrm{CHC}=$ contracetivo hormonal combinado; $\mathrm{DEXA}=$ dual-energy $\mathrm{X}$-rayabsorptiometry; $\mathrm{DMO}=$ densidade mineral óssea; $\mathrm{EE}=$ etinilestradiol; $\mathrm{NE}=$ nível de evidência.

ram em conjunto a inclusão/exclusão do artigo com uma taxa de concordância final de $100 \%$. A leitura integral foi realizada e a avaliação da qualidade e NE dos artigos incluídos discutida por todos os autores.

\section{RESULTADOS}

A pesquisa inicial identificou 76 artigos. Destes, foram incluídos no estudo cinco: dois ECAC, uma RS, um estudo de coorte e uma guideline. As restantes publicações foram excluídas, em conformidade com a metodologia definida anteriormente.

Em 2010, a Faculty of Sexual and Reproductive Health Care, baseando-se num estudo da The Cochrane Library de 2006, refere que os CHC parecem não afetar a DMO em mulheres em idade fértil (NE 3) (Quadro I).

Relativamente à RS de 2015, composta por 18 artigos, dois estavam direcionados para a população definida pelas investigadoras (Quadro II).

Lattakova e colaboradores, em 2009, concluíram que
( $n=73)$, CHC na dose de 30-35 $\mu \mathrm{g}(n=121)$ e sem CHC $(n=107)$. Este estudo demonstrou que a DMO na coluna lombar ou colo do fémur não foi significativamente diferente nos três grupos. A esta RS foi atribuído o nível de evidência 2.

Em 2011, Scholes e colaboradores realizaram um estudo de coorte com seguimento de 24 a 36 meses, que incluiu 301 adolescentes divididas em três grupos: $\mathrm{CHC}$ na dose $<30 \mu \mathrm{g}(n=73)$, CHC na dose de 30-35 $\mu \mathrm{g}(n=121)$ e sem CHC $(n=107)$.

Foi verificado que, ao longo dos 36 meses de estudo, a aquisição de DMO foi sempre superior no grupo sem CHC em comparação com os restantes grupos de tratamento. Até aos 24 meses, o grupo com dose superior de EE (30 a $35 \mu \mathrm{g}$ ) aumentou mais em relação à linha basal do que o grupo com dose inferior. Dos 24 aos 36 meses, o grupo a fazer CHC com EE com 30-35 $\mu$ g demonstrou um decréscimo na aquisição de DMO (descida de $1 \%$ em relação à linha basal). Neste mesmo pe- 


\begin{tabular}{|c|c|c|c|c|}
\hline Referência & População & Intervenção & Resultados & NE \\
\hline $\begin{array}{l}\text { Scholes, et } \\
\text { al. (2011) }\end{array}$ & $\begin{array}{l}\text { Adolescentes } \\
14-18 \text { anos } \\
(n=301)\end{array}$ & $\begin{array}{l}\text { A: } n=73 \text { com EE }<30 \mu \mathrm{g} \\
\text { B: } n=121 \text { com EE } 30-35 \mu \mathrm{g} \\
\text { C: } n=107 \text { sem CHC } \\
\text { Seguimento de } 36 \text { meses } \\
\text { com DEXA (corporal, coluna } \\
\text { lombar e colo do fémur) a } \\
\text { cada seis meses }\end{array}$ & $\begin{array}{l}\text { C: aumentou DMO corporal } 2,03 \%, 0,67 \% \text { no colo do fémur } \\
\text { e } 2,26 \% \text { na coluna lombar } \\
\text { A e B: benefício similar no colo do fémur, mas menor na } \\
\text { coluna lombar e corporal } \\
\text { Até } 24 \text { meses: o aumento de DMO em relação à linha basal } \\
\text { foi maior no grupo B do que no grupo A } \\
\text { Dos } 24 \text { aos } 36 \text { meses, o grupo B demonstrou um decréscimo } \\
\text { na aquisição de DMO (descida de } 1 \% \text { em relação à linha } \\
\text { basal) } \\
\text { Entre B e C verificou-se uma diferença ES (superior para C) } \\
\text { relativamente à DMO coluna (B 1,32\% vs C } 2,26 \% \text { ) e } \\
\text { corporal (B } 1,45 \% \text { vs C } 2,03 \% \text { ) }\end{array}$ & 2 \\
\hline
\end{tabular}

Legenda: $\mathrm{CHC}=$ contracetivo hormonal combinado; DEXA = dual-energy $X$-rayabsorptiometry; $\mathrm{DMO}=$ densidade mineral óssea; $\mathrm{EE}=$ etinilestradiol; $E S$ = estatisticamente significativo; $N E$ = nível de evidência.

ríodo, nas adolescentes que estavam a fazer um $\mathrm{CHC}$ com EE em dose inferior a aquisição de DMO manteve-se em progressão ascendente (NE 2) (Quadro III).

Cibula e colaboradores realizaram, em 2012, um ECAC com 84 adolescentes com idades compreendidas entre os 15 e os 19 anos, com desenho cruzado e ordem reversa após nove meses, totalizando um seguimento de 18 meses. Foram divididas em dois grupos: um grupo de 56 adolescentes utilizadoras de CHC com EE 30 $\mu \mathrm{g}$ durante nove meses, que depois passaram a utilizar EE com $15 \mu \mathrm{g}$; o segundo grupo era constituído por 28 mulheres sem método contracetivo hormonal. Foi realizada densitometria óssea (DEXA) aos 0, 9 e 18 meses. Concluiu-se que nas não utilizadoras, assim como nas mulheres com CHC com EE $30 \mu \mathrm{g}$, a DMO aumentou (aumento na coluna lombar, $p<0,05$ ). Nas mulheres com CHC com EE $15 \mu \mathrm{g}$ não se verificou um aumento da DMO (NE 2) (Quadro IV).

Gai e colaboradores realizaram, em 2012, um ECAC com 450 adolescentes que nunca tinham efetuado um CHC, com idades compreendidas entre os 16 e os 18 anos, avaliando a DMO por DEXA na coluna lombar (L2-L4), no colo femoral e corporal. Foram divididas em 3 grupos: um terço iniciou CHC com EE $30 \mu \mathrm{g}$, um terço iniciou CHC com EE $35 \mu \mathrm{g}$ e as restantes iniciaram contraceção não hormonal. Verificaram, após 24 meses, que no primeiro grupo houve um decréscimo de $0,3 \%$ e de $0,61 \%$ do aumento da DMO da coluna lombar e do colo do fémur, respetivamente; no segundo grupo houve um aumento de $0,3 \%$ e de $0,49 \%$ da DMO da coluna lombar e do colo do fémur, respetivamente; no terceiro grupo verificaram um aumento de $1,88 \% \mathrm{e}$ de $0,98 \%$ da DMO da coluna lombar e do colo do fémur, respetivamente. No entanto, não se verificaram diferenças estatisticamente significativas na DMO na coluna lombar e no colo do fémur entre os três grupos após os 24 meses (NE 1) (Quadro IV).

\section{CONCLUSÕES}

Os estudos incluídos neste trabalho apresentam diferenças de metodologia, nomeadamente no que diz respeito ao tempo de seguimento das adolescentes incluídas, o que poderá contribuir para a heterogeneidade dos resultados. Os estudos nesta área são escassos e os existentes têm uma amostra reduzida, sendo que a maioria destes não analisaram fatores importantes na variação da densidade mineral óssea nesta faixa etária, como é o caso da dieta alimentar ou do exercício físico. 


\begin{tabular}{|c|c|c|c|c|}
\hline Referência & População & Intervenção & Resultados & NE \\
\hline $\begin{array}{l}\text { Cibula, et al. } \\
\text { (2012) }\end{array}$ & $\begin{array}{l}\text { Adolescentes } \\
15-19 \text { anos } \\
(n=84)\end{array}$ & $\begin{array}{l}\text { A: } n=28 \text { com CHC (EE } 30 \mu \mathrm{g}) \\
\text { B: } n=28 \text { com CHC (EE } 15 \mu \mathrm{g}) \\
\text { C: } n=28 \text { sem CHC } \\
\text { Seguimento de } 18 \text { meses } \\
\text { DEXA aos } 0,9 \text { e } 18 \text { meses }\end{array}$ & $\begin{array}{l}\text { A e B: DMO não aumentou com exceção do } \\
\text { grupo A que aumentou na coluna lombar }(p<0,05) \\
\text { C: aumentou a DMO } \\
\text { O aumento da DMO foi mais prejudicado no } \\
\text { grupo B }(p<0,05)\end{array}$ & 2 \\
\hline $\begin{array}{l}\text { Gai, et al. } \\
\text { (2012) }\end{array}$ & $\begin{array}{l}\text { Adolescentes } \\
\text { 16-18 anos, que } \\
\text { nunca tinham } \\
\text { efetuado CHC } \\
(n=450)\end{array}$ & $\begin{array}{l}\text { A: } n=150 \text { com CHC (EE } 30 \mu \mathrm{g}) \\
\text { B: } n=150 \text { com CHC (EE } 35 \mu \mathrm{g}) \\
\text { C: } n=150 \text { contraceção não hormonal } \\
\text { Seguimento de } 24 \text { meses } \\
\text { DEXA coluna e colo do fémur aos } 0 \text {, } \\
12 \text { e } 24 \text { meses }\end{array}$ & $\begin{array}{l}\text { Ao fim de } 24 \text { meses: } \\
\text { A DMO diminuiu no grupo A, (coluna lombar } \\
0,3 \% \text { e colo do fémur } 0,61 \% \text { ) } \\
\text { No grupo B e C aumentaram ( } 0,3 \% \text { na coluna } \\
\text { lombar e } 0,49 \% \text { no colo do fémur, para o grupo } \\
\text { B; e } 1,88 \% \text { na coluna lombar e } 0,98 \% \text { no colo } \\
\text { do fémur para o grupo } C \text { ) } \\
\text { Não se verificou significância estatística entre } \\
\text { os três grupos }\end{array}$ & 1 \\
\hline
\end{tabular}

Legenda: $\mathrm{CHC}=$ contracetivo hormonal combinado; $\mathrm{DEXA}=$ dual-energy $X$-rayabsorptiometry; $\mathrm{DMO}=$ densidade mineral óssea; $\mathrm{EE}=$ etinilestradiol; $\mathrm{ES}=$ estatisticamente significativo; $\mathrm{DMO}=$ densidade mineral óssea; $\mathrm{GC}$ = grupo-controlo; $\mathrm{NE}$ = nível de evidência.

Por definição, são consideradas mulheres em idade fértil as mulheres com idades compreendidas entre os 15 e os 49 anos. A guideline da Faculty of Sexual and Reproductive Health Care, de 2010, ao sistematizar que os CHC parecem não afetar a DMO em mulheres em idade fértil, perde força de recomendação na medida em que não distingue as adolescentes das jovens adultas ou mesmo das mulheres pré-menopáusicas.

O estudo de coorte englobado, ao prolongar o seguimento das jovens até aos 36 meses, permitiu perceber que a contraceção com CHC com dose igual ou superior a $30 \mathrm{~g}$ EE poderá ser mais importante nos dois primeiros anos após o início da contraceção. Os restantes estudos analisados nesta revisão realçam o benefício na DMO da toma de CHC nos dois primeiros anos. No entanto, após os 24 meses, o benefício não é tão evidente.

Esta revisão baseada na evidência revelou que as adolescentes que não utilizam CHC têm um maior aumento da DMO comparativamente às que iniciam este fármaco antes dos dezanove anos. Nas que iniciam esta classe terapêutica antes da idade adulta, em doses de
30 a $35 \mu$ g de etinilestradiol, regista-se um ganho maior de massa óssea comparativamente a doses inferiores a $30 \mathrm{~g}$ (força de recomendação B).

\section{AGRADECIMENTOS}

Às orientadoras de formação específica em Medicina Geral e Familiar das autoras, pelo apoio científico prestado durante a realização desta revisão.

\section{REFERÊNCIAS BIBLIOGRÁFICAS}

1. World Health Organization. Young people's health: a challenge for society (report of a WHO Study Group on Young People and Health for All by the year 2000). Geneva:WHO; 1986. ISBN 9241207310

2. Avery L, Lazdane G. What do we know about sexual and reproductive health of adolescents in Europe. Eur J Contracept Reprod Health Care. 2010;15(Suppl. 2):S54-66.

3. Ott MA, Sucato GS. Contraception for adolescents. Pediatrics. 2014;134(4):e1257-81.

4. Cibula D, Skrenkova J, Hill M, Stepan JJ. Low-dose estrogen combined oral contraceptives may negatively influence physiological bone mineral density acquisition during adolescence. Eur J Endocrinol. 2012;166(6):1003-11.

5. Ziglar S, Hunter TS. The effect of hormonal oral contraception on acquisition of peak bone mineral density of adolescents and young women. J Pharm Pract. 2012;25(3):331-40. 
6. Boot AM, de Ridder MA, Pols HA, Krenning EP, de Muinck Keizer-Schrama SM. Bone mineral density in children and adolescents: relation to puberty, calcium intake, and physical activity. J Clin Endocrinol Metab. 1997;82(1):57-62.

7. Sabatier JP, Guaydier-Souquières G, Benmalek A, Marcelli C. Evolution of lumbar bone mineral content during adolescence and adulthood: a longitudinal study in 395 healthy females 10-24 years of age and 206 premenopausal women. Osteoporos Int. 1999;9(6):476-82.

8. Lu PW, Briody JN, Ogle GD, Morley K, Humphries IR, Allen J, et al. Bone mineral density of total body, spine, and femoral neck in children and young adults: a cross-sectional and longitudinal study. J Bone Miner Res. 1994;9(9):1451-8.

9. Committee on Gynecologic Practice. ACOG Committee Opinion Number 540: Risk of venous thromboembolism among users of drospirenone-containing oral contraceptive pills. Obstet Gynecol. 2012;120(5):1239-42.

10. Sociedade Portuguesa de Ginecologia, Sociedade Portuguesa da Contracepção, Sociedade Portuguesa de Medicina da Reprodução. Consenso sobre Contracepção (2011). Lisboa: Frist News; 2011.
11. Practice Committee of the American Society for Reproductive Medicine. Combined hormonal contraception and the risk of venous thromboembolism: a guideline. Fertil Steril. 2017;107(1):43-51.

12. Castro J, Tavares B, Guedes M. Contraceção hormonal combinada num grupo de adolescentes. In: XXIV Encontro do Internato de Medicina Geral e Familiar da Zona Norte, Penafiel, 16-17 de novembro de 2017. Poster.

\section{CONFLITO DE INTERESSES}

Os autores declaram não ter conflitos de interesses.

\author{
ENDEREÇO PARA CORRESPONDÊNCIA \\ Juliana Silva Castro \\ E-mail: jsc.fmuc@gmail.com \\ https://orcid.org/0000-0002-3052-5743
}

Recebido em 21-09-2017

Aceite para publicação em 26-10-2018

\section{ABSTRACT}

\section{EFFECTS OF ETHINYLESTRADIOL CONTRACEPTION IN BONE MINERAL DENSITY IN ADOLESCENT WOMEN: WHAT IS THE EVIDENCE?}

Objective: Combined hormonal contraceptives ( $\mathrm{CHC}$ ) are the most used hormonal contraception method in adolescence. They lead to lower levels of estradiol than physiological ones, which may influence the acquisition of peak bone mineral density (BMD). The objective of this study was to determine the impact of CHC with 30-35 $\mathrm{\mu g}$ of ethinyl estradiol (EE) on BMD when compared to placebo or lower doses of EE in healthy female adolescents between 10-19 years old.

Sources: National Guideline Clearinghouse, National Institute for Health and Care Excellence Guidelines Finder, Canadian Medical Practice Guidelines Infobase, The Cochrane Library, Database of Abstracts of Reviews of Effectiveness, Bandolier, Evidence-Based Medicine Online, and MEDLINE.

Methods: We searched clinical guidelines, randomized controlled trials, systematic reviews and meta-analysis, published between March 2007 and March 2017 in Portuguese, Spanish, English or French. The MESH terms used were: 'adolescent', 'ethinyl estradiol' and 'bone density'. To stratify the level of evidence and the strength of recommendation the Strenght of Recommendation Taxonomy, of the American Academy of Family Physicians was used.

Results: Seventy-six publications were identified, of which five were included: two randomized controlled trials, one systematic review, one cohort study, and one guideline. The first showed that non-CHC users' adolescents have higher BMD than those who use $\mathrm{CHC}$, regardless of drug dosage. The BMD of adolescents using a higher dosage of ethinyl estradiol (30-35 $\mu \mathrm{g})$ appeared to be less affected than those with a lower dosage $(<30 \mu \mathrm{g})$. In the included systematic review discordant data were found, with one of the studies reporting no differences between the various doses of EE. The included cohort study revealed an

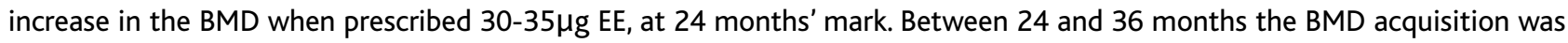

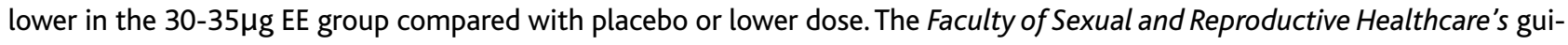
deline reports that dosage does not appear to affect BMD.

Conclusion: In teenagers, the use of ethinyl estradiol at a dosage equal or higher to 30ug, for a lower impact on BMD, has received a Grade B Recommendation.

Keywords: Adolescent; Ethinyl estradiol; Bone mineral density. 